\title{
A SURVEY ON UNDERWATER SENSOR NETWORKS
}

\author{
Rajshekhar S A ${ }^{1}$, Dr Arun Biradar ${ }^{2}$ \\ ${ }^{1}$ Associate Professor EWIT, India, rajshekharsa@ewit.edu, \\ ${ }^{2}$ Professor and HOD Department of Computer Science and engineering EWIT, India, hodcsea@ gmail.com
}

\begin{abstract}
Wireless sensor networks (WSN) behave as a digital skin, providing a virtual layer where the information about the physical world can be accessed by any computational system. In our earth $75 \%$ covered by water that could be river and ocean also. The underwater sensor network are enabling technology and become more and more popular for monitoring vast area of oceans. Underwater sensor Networks consist of a variable number of sensors that are deployed to perform monitoring tasks over a given area. The UWSNs provide continuous monitoring for various applications like pollution monitoring, submarine detection, disaster prevention etc.
\end{abstract}

In this paper discuss an invaluable resource for realizing the vision of the Internet of Things (IoT). we will focus on the issues that take place at the network level and applications and challenges of underwater sensor, we also discuss the main problem or issue in underwater sensor network.

Key words : Internet of Things; Internet; UWSN, UWASNs.

\section{INTRODUCTION}

In the upcoming Internet of Things (IoT), the everyday objects that surround us will become proactive actors of the Internet, generating and consuming information. The elements of the IoT comprise not only those devices that are already deeply rooted in the technological world (such as cars or fridges), but also objects foreign to this environment (garments or perishable food), or even living beings (plantations, woods or livestock). By embedding computational capabilities in all kinds of objects and living beings, it will be possible to provide a qualitative and quantitative leap in several sectors: healthcare, logistics, domotics, entertainment, and so on. In fact, one of the most important elements in the IoT paradigm is wireless sensor networks (WSN). The benefits of connecting both WSN and other IoT elements go beyond remote access, as heterogeneous information systems can be able to collaborate and provide common services. This integration is not mere speculation, but a fact supported by several international companies.

Oceans represent more than $2 / 3$ of the Earth's surface. Underwater wireless sensor networks (UWSNs) have gained the attention of the scientific and industrial communities due their potential to monitor and explore aquatic environments. UWSNs have a wide range of possible applications such as to monitoring of marine life, pollutant content, geological processes on the ocean floor, oilfields, climate, and tsunamis and seaquakes; to collect oceanographic data, ocean navigation assistance, in addition to being utilized for tactic surveillance applications Networks of sensors and AUVs, such as the Odyssey-class AUVs, can perform synoptic, cooperative adaptive sampling of the 3D coastal ocean environment.

Experiments such as the Monterey Bay field experiment demonstrated the advantages of bringing together sophisticated new robotic vehicles with advanced ocean models to improve the ability to observe and predict the characteristics of the oceanic environment[1].

\section{A. Applications of Underwater Sensor Networks}

- Environmental monitoring-UW-ASNs can perform pollution monitoring (chemical, biological and nuclear). For example, it may be possible to detail the chemical slurry of antibiotics, estrogen-type hormones and insecticides to monitor streams, rivers, lakes and ocean bays (water quality in situ analysis). Monitoring of ocean currents and winds, improved weather forecast, detecting climate change, under-standing and predicting the effect of human activities on marine ecosystems, biological monitoring such as tracking of fishes or micro- organisms, are other possible applications. For example, the design and construction of a simple underwater sensor0020network is described to detect extreme temperature temperature gradients (thermoclines), which are considered to be a breeding ground for certain marine micro-organisms[1].

- Undersea explorations-Underwater sensor networks can help detecting underwater oilfields or reservoirs, determine routes for laying undersea cables, and assist in exploration for valuable minerals. 
- Disaster prevention- Sensor networks that measure seismic activity from remote locations can provide tsunami warnings to coastal areas, or study the effects of submarine earthquakes (seaquakes).

Assisted navigation-Sensors can be used to identify hazards on the seabed, locate dangerous rocks or shoals in shallow waters,

mooring positions, submerged wrecks, and to perform bathymetry profiling.

- Distributed tactical surveillance- AUVs and fixed underwater sensors can collaboratively monitor areas for surveillance, reconnaissance, targeting and intrusion detection systems. For example, a 3D underwater sensor network is designed for a tactical surveillance system that is able to detect and classify submarines, small delivery vehicles (SDVs) and divers based on the sensed data from mechanical, radiation, magnetic and acoustic microsensors. With respect to traditional radar/sonar systems, underwater sensor networks can reach a higher accuracy, and enable detection and classification of low signature targets by also combining measures from different types of sensors.

\section{B. Major challenges in design of USNs are}

- The available bandwidth is severely limited

- The underwater channel is severely impaired, especially due to multi-path and fading;

- Propagation delay in underwater is five orders of magnitude higher than in radio frequency (RF) terrestrial channels, and extremely variable;

- High bit error rates and temporary losses of connectivity (shadow zones) can be experienced, due to the extreme characteristics of the underwater channel;

- Battery power is limited and usually batteries cannot be recharged, also because solar energy cannot be exploited;

- Underwater sensors are prone to failures because of fouling and corrosion.

\section{LITERATURE REVIEW}

The traditional approach for ocean-bottom or ocean-column monitoring is to deploy underwater sensors that record data during the monitoring mission, and then recover the instruments[2].

This approach has the following disadvantages:

- No real-time monitoring- The recorded data cannot be accessed until the instruments are recovered, which may happen several months after the beginning of the monitoring mission. This is critical especially in surveillance or in environmental monitoring applications such as seismic monitoring[3].

- No on-line system reconfiguration-Interaction between onshore control systems and the monitoring instruments is not possible. This impedes any adaptive tuning of the instruments, nor is it possible to reconfigure the system after particular events occur.

- No failure detection. If failures or mis-configurations occur, it may not be possible to detect them before the instruments are recovered. This can easily lead to the complete failure of a monitoring mission[3].

- Limited storage capacity- The amount of data that can be recorded during the monitoring mission by every sensor is limited by the capacity of the onboard storage devices (memories, hard disks)[4].

\section{UNDERWATER SENSOR NETWORK ARCHITECTURE}

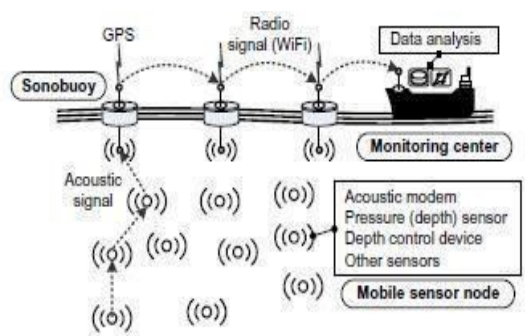

Figure 1 : SEA swarm Architecture

Underwater sensor networks were recently proposed to support time-critical aquatic applications such as submarine tracking and harbor monitoring. Unlike traditional tethered sensors, a large number of underwater mobile sensor nodes are dropped to the venue of interest to form a SEA Swarm (Sensor Equipped Aquatic Swarm) that moves as a group with water current. Each sensor is equipped with a low bandwidth acoustic modem and with various sensors (e.g., Drogues). Moreover, it can control its depth through a fish-like bladder apparatus and a pressure gauge. The swarm is escorted by sonobuoys at the sea surface that are equipped with both acoustic and radio (e.g., WiFi or Satellites) communications and GPS (See Figure 1).

There are several major advantages of SEA swarm architecture. First, mobile sensors provide 4D (space and time) monitoring, thus forming dynamic monitoring coverage. Second, the multitude of sensors (as in the SEA swarm) help provide extra control on redundancy and granularity. Third, floating sensors increase system re-configurability because they can control their depth; 
moreover they resurface once depleted of energy and can thus be recovered and reused[5].

In the SEA swarm architecture, each sensor monitors local underwater activities and reports time-critical data to any one of the sonobuoys using acoustic multi-hopping; then the data are delivered to a monitoring center using radio communications. The main focus of this paper is to design an efficient anycast routing protocol from a mobile sensor to any one of the sonobuoys on the sea level. However, this is challenging due to node mobility and limited resources (bandwidth and energy) of mobile sensors. An underwater acoustic channel has low bandwidth and propagation latency five orders of magnitude higher than the radio channel[5].

Acoustic transmissions consume much more energy than terrestrial microwave communications. Such severe limitations in communication bandwidth coupled with high latency and limited energy make the network vulnerable to congestion due to packet collisions. Under these circumstances, minimizing the number of packet transmissions is important for at least two reasons: minimizing congestion and minimizing energy consumption.

Conventional proactive/reactive routing protocols (e.g., OLSR, AODV, etc.) rely on systematic flooding for route discovery and maintenance, potentially causing excessive energy consumption and collisions. In a SEA swarm scenario, general 3D geographic routing is preferable as it is stateless. However, geographic routing requires online, distributed localization of mobile sensors which is expensive and takes a long time to converge. Also, Durocher et al showed that efficient recovery from a local maximum may not always be feasible in 3D geographic routing, thus requiring an expensive exhaustive search such as $3 \mathrm{D}$ flooding and random walks[5].

\section{4 . PROBLEM IN UNDERWATER SENSOR NETWORK}

A. Costly devices - Underwater sensor devices are more costly. And no more supplier are provides these such kind of devices because these are devices are part of research oriented activity. Underwater sensor devices are not easily available in the market[2].

B. Hardware Protection requirement -The underwater devices is more expensive. So device protection or hardware protection is required against water[2].

C. Needed high power for communication- In underwater sensor communication require more power because the data transfer will done in water medium.So,in water more electricity is require for data exchanging. Communication among UWSNs is probably the biggest challenge facing UWSNs. point out that path loss (attenuation and geometric spreading), noise (man-made and ambient), multi-path, high propagation delays, and Doppler spread, can significantly disrupt or degrade the underwater communication channel. Another problem is that standard acoustic transducers cannot simultaneously transmit and receive. Underwater network communications are therefore always halfduplex[3].

G. Propagation delay- The propogation delay is major problem in underwater sensor network. The propogation of accoustic channels in underwater is order of magnitude higher than radio frequency in terrestrial sensor network.[3]

H. Localization- Localization means find the location of sensor in underwater sensor network.So,localization is another major problem yet to be solved. Localization is the challenging factor that is require for data labeling while some time critical applications require data without time delay.

Limited battery power- UWSN lifetime is an area of extensive research. UWSNs suffer from a sensor's fouling and corrosion Electronics components, such as the battery, tend to degrade faster under extremely low temperatures such as the one found in deep underwater. As a consequence, the USWN lifetime is much shorter than the lifetime of a comparable TWSN. In underwater sensor battery has limited power. A shorter lifetime increases the replacement costs because the underwater sensor battery is not chargable.

J. Bandwidth size limitation- In the underwater sensor network bandwidth is another big problem. Because bandwidth size is limited.

K. Reliability - This is one of the major design issues for reliable delivery of sensed data to the surface sink or water surface is a challenging task compare to forwarding the collected data to the control center or on- shore station.

F. Temporary losses- Temporary losses means the packet losses when connectivity time and packet sending time[4].

\section{CONCLUSION}

In this paper introduced the underwater sensor network. Present the main application of underwater sensor network. In this paper also introduced the architecture of underwater sensor network, routing family and main challenges of underwater sensor network.

\section{REFERENCES}

[1] I. F. Akyildiz, D. Pompili, and T. Melodia, "Underwater acoustic sensor networks: Research challenges," Ad

Hoc Netw., vol. 3, no. 3, pp. 257-279, 2005.

https://doi.org/10.1016/j.adhoc.2005.01.004

[2] I. Vasilescu, K. Kotay, D. Rus, M. Dunbabin, and P. Corke, "Data collection, storage, and retrieval with an underwater sensor network," in Proc. $3^{\text {rd }}$ 
Rajshekhar S A et al., International Journal of Computing, Communications and Networking, 7(2) April - June 2018, 310-313

ACM Int. Conf. Embedded Netw. Sensor Syst., 2005, pp.

$154-165$.

https://doi.org/10.1145/1098918.1098936

[3] J. Partan, J. Kurose, and B. N. Levine, "A survey of practical issues in underwater networks," in

Proc. 1st ACM

https://doi.org/10.1145/1161039.1161045

[4] M. Stojanovic and J. Preisig, "Underwater acoustic

communication channels: Propagation models and statistical characterization," IEEE Commun. Mag., vol. 47, no. 1, pp.

84-89, Jan. 2009.

https://doi.org/10.1109/MCOM.2009.4752682

[5] U. Lee, P. Wang, Y. Noh, L. F. M. Vieira, M. Gerla, and J.-H. Cui, "Pressure routing for underwater sensor networks," in Proc. IEEE

INFOCOM, 2010, pp. 1-9.

https://doi.org/10.1109/INFCOM.2010.5461986 